\title{
Inferior Vena Cava Injuries: A case series and review of the South African experience
}

\author{
P.L. van Rooyen, V.O.L. Karusseit, T.R. Mokoena \\ Department of Surgery, University of Pretoria and Kalafong Hospital
}

\begin{abstract}
Introduction

Penetrating injury may involve the major vessels in the abdomen. Injury to the abdominal inferior vena cava (IVC) is uncommon and is usually caused by gunshot wounds. Mortality from IVC injuries is high and has changed little over time.
\end{abstract}

Aim

The aim of the study was to report a series of IVC injuries from an urban trauma unit and to compare this with reports from similar institutions.

Method

A retrospective review of penetrating abdominal injuries at Kalafong Hospital from 1993 to 2010 was performed. All cases of injury to the IVC were retrieved and the following data recorded: patient demographics, incident history, origin of referral, description of the IVC injury, associated injuries, operative management, hospital stay and outcome. The results were compared to those from similar institutions.

Results

Twenty seven patients with IVC injuries were treated. All were caused by gunshot wounds, and all had associated intra-abdominal injuries. The majority (56\%) of injuries were infrarenal. The injury was managed most commonly by venorrhaphy and, when successful, all the patients survived. A third of patients with infrarenal injuries died, some after exploration of a stable peri-caval haematoma. Ten of the patients died (37\%), half of them during surgery. These results are similar to those from similar institutions from earlier time periods.

Conclusions

This report concurs with other studies. IVC injury carries a high mortality rate and that this has not improved over several decades. Less aggressive management of some stable patients or stable injuries is proposed by the authors for possible improvement of the mortality rate.

Keywords: Inferior vena cava; Abdominal trauma; Penetrating injury; 


\section{Background}

Penetrating abdominal trauma is a certain component of the practice of the urban trauma unit. Knives are ubiquitous and handguns are readily available in many countries. The large vessels in the abdomen are uncommonly involved in such injuries. Most injuries to the IVC are due to gunshots. Injury to the abdominal inferior vena cava (IVC) has a peculiar presentation and requires specific surgical management. This is because of the low intraluminal pressure of the IVC and its relatively thin wall. In addition, the superior part of the IVC is located close to its final ingress to the heart and is relatively inaccessible to the surgeon. Injuries to the IVC carry a high mortality rate, ranging up to $65 \% .1$ This is partly due to associated injuries. Approximately $30 \%-50 \%$ of patients do not reach a hospital, and about $30 \%$ of those that do, die as a result of their injuries. 2 The mortality rate of IVC injuries has changed little over time and surgical approaches seem to have stagnated.3 Reports on management of these injuries remain important in order to discern any possible improvement in logistical or surgical management.

\section{Aim}

The aim of this study was to report on the experience of an urban trauma unit in the management of penetrating IVC injuries, and to compare this with reports from similar institutions.

\section{Setting}

Kalafong Hospital is a regional general hospital situated in The Tshwane Metropolitan region and forms part of the training platform for the University of Pretoria. It has a level II trauma service.

\section{Patients and Methods}

A retrospective review was performed of abdominal injuries treated at Kalafong Hospital in which the IVC was involved. The study spanned a period of 17 years from 1993 to 2010 . The departmental records database was searched for cases of trauma laparotomy, as well as for the ICD 9 code for IVC injury, 902.1. Data were collected regarding patient demographics, incident history, origin of referral, description of the IVC injury, associated injuries, operative management, hospital stay and patient outcome. Initial management was according to standard Advanced Trauma and Life-Support (ATLS) guidelines. The decision to perform a laparotomy was based on haemo-dynamic instability despite, or after, initial fluid resuscitation, and/or signs of peritonism. No preoperative imaging studies were performed. Initial management of the patients was by surgical registrars. Consultants were called when registrars found this necessary and this occurred intra-operatively in most cases. Access to the abdomen was through a long midline incision. In cases with active haemorrhage from the retroperitoneum control was attempted with direct pressure to the area of injury, which was then explored. In addition, all zone 1 retroperitoneal haematomas were explored, as per departmental protocol. Operative procedures on the IVC were venorrhaphy when possible, or 
ligation. Uncontrollable bleeding was managed by packing. Patients were managed postoperatively in the intensive care unit whenever possible, beds in the ICU being at a premium.

\section{Results}

Twenty seven patients with IVC injuries were treated during the study period, representing $0.5 \%$ of trauma laparotomies. There were 24 males and 3 females with an average age of 30.6 years, 22 (81\%) being below the age of 40 years. Nine of the patients $(33 \%)$ had been transferred from peripheral hospitals - all by road ambulance, for distances of 50 to 200 kilometres. All injuries had been caused by shots from handguns. It was not usually possible to obtain information regarding the calibre of the guns in most cases. Entrance wounds were in the epigastrium in 17 cases, the back 5 cases and the chest in 4 cases.

There were associated intra-abdominal injuries in all cases. All patients sustained hollow visceral injuries: small intestine in 16 (64\%) and colon in 10 (40\%). Solid organ injuries occurred in 14 (52\%) of patients, being mainly to the liver in 11 patients (41\%), but also the pancreas and kidneys. Six patients (22\%) had associated major vascular injuries.

Table 1. Mortality in relation to the operative management of IVC injuries in 27 patients.

\begin{tabular}{|c|c|c|}
\hline Procedure & Number & Mortality $\mathrm{n}(\%)$ \\
\hline Venorrhaphy & 14 & $0(0)$ \\
\hline Venorrhaphy and packing & 2 & $1(50)$ \\
\hline Ligation & 4 & $2(50)$ \\
\hline Attempted control / exsanguination & 5 & $5(100)$ \\
\hline Packed & 1 & $1(100)$ \\
\hline Synthetic graft repair & 1 & $1(100)$ \\
\hline Total & 27 & $10(37)$ \\
\hline
\end{tabular}

The surgical procedures performed are shown in table 1 . Venorrhaphy was performed most commonly. In 4 cases the IVC was ligated below the renal veins. Interposition synthetic grafting was performed in 1 case for a suprarenal injury. Packing only was performed in 2 patients with associated liver injuries, and in one other patient.

The anatomical distribution of the IVC injuries is shown in table 2 together with the mortality for each region. Injuries were classified as infrarenal, suprarenal and retrohepatic. The majority (56\%) of the injuries were infrarenal, and these patients had the best survival. Nevertheless, 5 of the 15 patients $(33 \%)$ died. The management and mortality rates of the infrarenal injuries are shown in table 3. 
Table 2. Mortality in relation to the site of IVC injury in 27 patients.

\begin{tabular}{lcc} 
Site of injury & Number & Mortality $\mathrm{n}(\%)$ \\
\hline Retrohepatic & 3 & $3(100)$ \\
Suprarenal & 9 & $2(22)$ \\
Infrarenal & 15 & $5(33)$ \\
Total & 27 & $10(37)$ \\
\hline
\end{tabular}

Table 3. Management and mortality in infrarenal IVC injuries in 15 patients.

\begin{tabular}{lcl} 
Procedure & Number (\%) & Mortality: Number (\%) \\
\hline Venorrhaphy & $8(53)$ & 0 \\
Venorrhaphy + packing & $1(7)$ & $1(100)$ \\
Ligation & $4(29)$ & $2(50)$ \\
Failed control & $2(14)$ & $2(100)$ \\
Total & 15 & $5(33)$ \\
\hline
\end{tabular}

The overall mortality in this series was $37 \%$ (10 of 27 patients). Half of the deaths occurred during surgery and the rest postoperatively in the ICU, except for 1 late death from sepsis after 77 days. The three patients with retro-hepatic IVC injuries all died, 2 intra-operatively and 1 later in the ICU. Five of the six cases with other major vascular injuries died. The only survivor was a patient who had concomitant injuries to the common iliac veins. These were sutured and the IVC ligated. In cases where venorrhaphy alone could be performed successfully all the patients survived. These were all infrarenal injuries. Four of the 9 patients transferred from distant hospitals died (44\%). However 2 had retrohepatic injuries and 1 a suprarenal injury. The other patient exsanguinated in theatre from an infrarenal injury after a $200 \mathrm{~km}$ road transfer. All the patients who were discharged from the ICU survived. The average hospital stay for patients who survived was 13.9 days.

\section{Discussion}

This report from an urban regional hospital shows a great deal of consistency with similar reports in the literature. Four previous reports from South Africa are of similar size but from different time periods. Two reports from Durban were on 28 patients in the 1980's and 26 patients in the 1990 's.4,5 The report from Baragwanath Hospital in Soweto in the 1990's was on 74 patients.6 The current study and the one from Cape Town7 straddled the two centuries. The 5 studies are compared in table 4.

Remarkably, the current series contains only cases of gunshot injuries. No IVC stab wounds or blunt injuries were managed during this period. The earlier series from Durban contains $53 \%$ stab wounds. 4 The proportion of gunshot wounds to the IVC in these reports has clearly increased over time indicating that handguns have become more freely available (Table 4). Nevertheless, gunshot wounds predominate in reports of the IVC injury, irrespective of era or origin (reviewed by Huerta et al)2. More recently, cases of IVC injury for inclusion in this study have all but dried up because there 
has been a dramatic decrease in penetrating injury due to interpersonal violence in our practice.

The period spanned by this study was characterised by a virtual epidemic of gunshot wounds. This is apparent also in the other South African studies from the 1900's and early part of this century, which show a predominance of gunshot wounds (Table 4).

Table 4. Comparison of 5 South African series of IVC injuries.

\begin{tabular}{|c|c|c|c|c|c|}
\hline $\begin{array}{l}\text { Origin } \\
\text { Period }\end{array}$ & $\begin{array}{l}\text { Durban }^{1} \\
1980-83\end{array}$ & $\begin{array}{c}\text { Johannesburg }^{2} \\
1990-94^{*}\end{array}$ & $\begin{array}{l}\text { Durban }^{1} \\
1990-95\end{array}$ & $\begin{array}{c}\text { Cape Town } \\
1999-2003\end{array}$ & $\begin{array}{c}\text { Pretoria }^{4} \\
1993-2005\end{array}$ \\
\hline Patients: number & 28 & 74 & 26 & 48 & 26 \\
\hline Males n $(\%)$ & $26(93)$ & $69(93)$ & $24(92)$ & $42(86)$ & $23(88)$ \\
\hline Average age (years) & 30 & 31 & 29 & 27 & 31 \\
\hline $\begin{array}{l}\text { Associated } \\
\text { Injuries } n(\%)\end{array}$ & & & & & \\
\hline Liver & $15(53)$ & $21(28)$ & $9(34)$ & $22(46)$ & $11(41)$ \\
\hline Small Bowel & $8(28)$ & $38(57)$ & $8(30)$ & $23(48)$ & $14(54)$ \\
\hline $\begin{array}{l}\text { Colon } \\
\text { Anatomic }\end{array}$ & $7(25)$ & $7(9)$ & $8(30)$ & $15(31)$ & $10(38)$ \\
\hline location n(\%) & & & & & \\
\hline Retrohepatic & $7(25)$ & $14(19)$ & $3(11)$ & $1(2)$ & $3(12)$ \\
\hline Suprarenal & $13(46)$ & $17(22)$ & $8(30)$ & $6(13)$ & $9(36)$ \\
\hline Infrarenal & $8(28)$ & $43(59)$ & $15(55)$ & $41(85)$ & $15(56)$ \\
\hline & & & & & \\
\hline Gunshot & $7(33)$ & $67(91)$ & $17(65)$ & $45(94)$ & $27(100)$ \\
\hline Stab & $15(53)$ & $7(9)$ & $5(19)$ & $1(2)$ & 0 \\
\hline Blunt & $4(14)$ & - & $4(16)$ & $2(4)$ & 0 \\
\hline $\begin{array}{l}\text { Mortality } \\
\text { (overall) } \mathrm{n}(\%)\end{array}$ & $10(36)$ & $32(43)$ & $23(88)$ & $15(31)$ & $10(37)$ \\
\hline
\end{tabular}

* Penetrating injuries only

Hospitals: 1 = King Edward VIII, 2 = Baragwanath, 3 = Groote Schuur, 4 = Kalafong

The reports from South Africa all show the same predominance of young males and anatomical distribution of IVC injuries, except for the report from Cape Town in which $85 \%$ of the injuries were infrarenal. The current series shows a greater proportion of associated liver and small bowel injury.

The mortality rate of $88 \%$ was unusually high in the later report from Durban. This could have been due to a twofold increase in firearm injuries compared to the first publication from that institution. In a report from probably similar circumstances in Pakistan, the mortality rate was also high, viz $63 \% .8$ The mortality rates in the other South African series range from 31 to $40 \%$. The mortality of different institutions cannot readily be compared because the rate is dependent on several factors. The more efficient the system for transporting trauma cases to a hospital, the greater the proportion of more serious cases that will be managed at that facility, and therefore the higher the mortality rate.2 Likewise the higher mortality rate for the later report from Durban could reflect improved rapid transport for the casualties than in the earlier series. This is most likely the explanation for mortality rates of more than $50 \%$ from some centres with highly-developed infrastructures.1,9 A 
greater proportion of patients in the latter reports will have been in extremis due to serious injury whereas $35 \%$ of patients in the present series survived prolonged transfer, indicating less serious and probably tamponaded injury of the IVC.

Several factors that have been shown to predict poor survival are apparent also in the present series. All patients with retrohepatic IVC injuries died of exsanguination during surgery. Similar results are reported by most authors.1,2,6 The series from Cape Town included only 1 retrohepatic injury, and a somewhat better survival rate.7 In the current report 5 of the 6 patients with other major vascular injuries died. Poor outcome in these patients is also reported by other authors.3,9 Associated injuries of the aorta are particularly devastating.

Survival was $100 \%$ in patients with infrarenal injuries that could be repaired primarily. Similar findings have been reported previously by other authors.1,2 This is probably due to ease of accessibility of the injury and of control of bleeding in these cases.

Five of the 15 patients (33\%) with infrarenal injuries in this series died, 2 after ligation and 3 after attempted venorrhaphy (Table 3 ). This is probably indicative of futile attempts at repair in patients that would have benefited from curtailing the procedure earlier. It is important to avoid the "lethal triad" of acidosis, hypothermia and coagulopathy induced by prolonged shock.7 The object of surgery should be to stop the bleeding and not to achieve a patent, undistorted IVC. Injudicious complex repairs with venous patching or prosthetic grafting should be avoided when simple ligation of the IVC would save the patient's life. Ligation carried a 50\% mortality in the present study ( 2 of 4 patients). Little can be concluded from so few patients but earlier ligation during the procedure might have been beneficial.

Packing of the injury was employed in only 3 of the cases in this study, 2 after venorrhaphy. Two of these patients died indicating that packing was a last ditch manoeuvre. Packing might have benefitted more patients given that several died of exsanguination during attempted control (Table $1)$.

A tamponaded or contained haematoma has been shown to be a beneficial finding in IVC injuries.10 In several cases in this report such a haematoma was disturbed and the IVC exposed. This could partially explain the mortality of $33 \%$ in cases of otherwise usually favourable infrarenal injuries in this series. Conservative and even non-operative management has been advocated in cases of contained haematomas around the IVC. This approach would be especially appropriate in the less accessible suprarenal and retrohepatic injuries.11 The low pressure in the IVC and its retroperitoneal location probably favours sealing of the injury without pseudoaneurysm formation. 
Indeed, it is probable that some IVC injuries are missed and never subjected to surgery without any consequences.

A conservative operative approach during is obviously not without dangers, especially if a consultant is not involved in the surgery. Injury to other retroperitoneal organs may be missed. The accepted indications for exploration of zone 1 supramesocolic injuries must still be applied viz an expanding or pulsatile haematoma, retroperitoneal air bubbles or a fluid collection, especially if bile-stained. Similar caveats apply to a non-operative approach based on computed tomographic scan findings.

Recently endovascular procedures have been employed during management of IVC injuries. Bui and Mills were able to control active haemorrhage from the IVC by endovascular placement of a balloon catheter during surgery. Angeles et al achieved the same by endovascular placement of 2 balloons during surgery, one positioned cranially and one caudally to the injury. Castelli et al curtailed active haemorrhage from an IVC injury by emergency placement of an endovascular stent. These cases illustrate the great facility of endovascular procedures to manage major vessel disease, and will no doubt play an increasing role in IVC injury. The technology is, however, not universally available as is the case at our hospital.

The 5 series from South Africa span a quarter of a century. Apart from the one report from Durban, the mortality has remained constant in the region of $30-40 \%$. This is disappointing. One could speculate about the reasons for this, but the reality is that the same lack of improvement in the mortality of IVC injuries is apparent in the developed world. Paul et al report that significant improvement in logistics and staffing level did not improve mortality over a period of 20 years in their level I trauma unit. 3 They suggest that there is a category of patients that should possibly not be treated because of futility.

We would suggest that there is a category of patient at the opposite pole that should be treated conservatively, despite evidence of major intra-abdominal venous injury. This is the stable patient without peritonitic signs. This approach is widely applied in other circumstances, such as liver, renal and splenic injury, as well as for abdominal gunshot wounds.12 In the era of sophisticated imaging, even a demonstrated pericaval haematoma should not be subjected to surgery if there are no other reasons to operate.13 Such a case is described by Khan et al.14 A suggested algorithm illustrating this approach is depicted in figure 1 . This approach would avoid in addition unnecessary laparotomies, which carry a considerable morbidity rate.15 


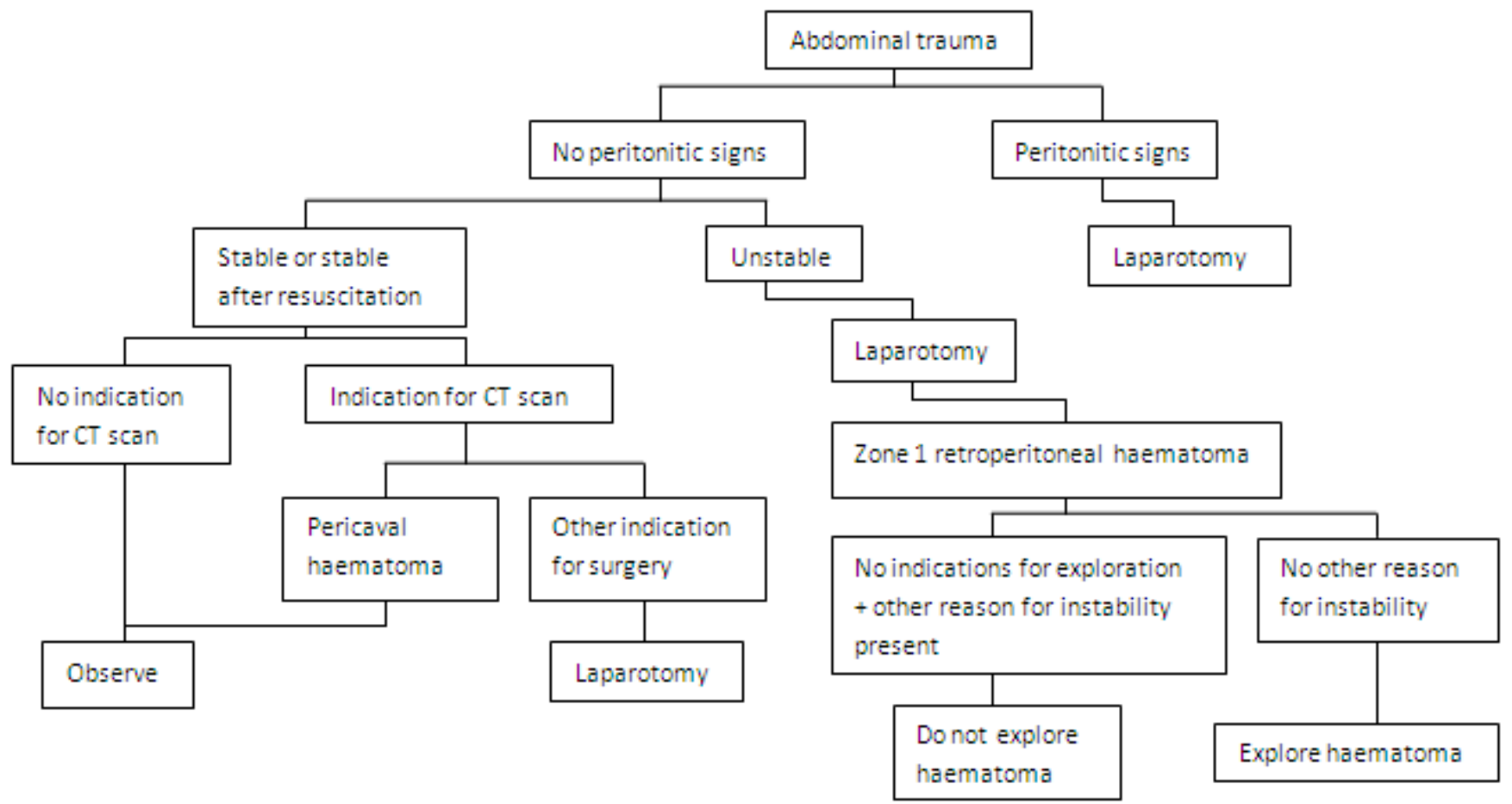

Figure 1. A suggested algorithm for the management of abdominal trauma and possible injury of the IVC.

It seems that more careful and judicious intra-operative management should also be part of surgical teaching. If there is no compelling reason to explore a "central" retroperitoneal or retrohepatic haematoma, it should probably be left undisturbed. This advice is not new.16 Nevertheless, inexperienced and over-enthusiastic trainees should be cautioned about the dangers of unnecessary exposure of the IVC.

\section{Summary}

Major IVC injuries may be associated with fatal outcome. Most of these patients would not have reached the hospital alive but for improved modern casualty evacuation logistics. Those patients whose IVC is contained in a haematoma warrant less aggressive surgical management. When laparotomy is indicated for reasons other than circulatory collapse, a contained IVC injury should not be disturbed. If haemorrhage from an IVC injury is found, surgeons should not hesitate to ligate the IVC, especially in infrarenal injuries, in order to curtail the operative time as part of "damage control" and avoid exsanguination or hypothermia and irreversible shock. 


\section{References}

1. Hansen CJ, Bermadas C, Wiest MA et al. Abdominal vena caval injuries: Outcomes remain dismal. Surgery 2000; 128(4): 572-578.

2. Huerta S, Bui TD, Nguyen TH et al. Predictors of mortality and management of patients with traumatic inferior rena cava injuries. The American Surgeon 2006; 72(4): 290-6.

3. Paul JS, Webb TP, Aprahamian C et al. Intraabdominal vascular injury: Are we getting any better? J Trauma, Injury, Infection and Critical Care 2010; 69(6): 1393-7.

4. Robbs JV, Costa M. Injuries to the great veins of the abdomen. S Afr J Surg 1984; 22(4): 223-

8.

5. Clarke DL, Madiba TE, Muckart DJ. Inferior vena caval injury in the firearm era. S Afr J Surg 1999; 37(4): 107-9.

6. Degiannis E, Velmahos GC, Levy RD et al. Ann R Coll Surg Engl 1996; 78: 485-9.

7. Navsaria PH, de Bruyn P, Nicol AJ. Penetrating abdominal vena cava injuries. Eur J Vasc Eudovasc Surg 2005; 30: 499-503.

8. Jan WA, Samad A, Anwar R. Mortality and morbidity of abdominal inferior vena-caval injuries. J Coll Physicians Surg Pak 2004; 14(10): 622-5.

9. Asensio JA, Chahwan S, Hanpeter D et al. Operative management and outcome of 302 abdominal vascular injuries. Am J Surg 2000; 180: 528-34.

10. Kuehne J, Frankhouse J, Modrall G et al. Determinants of survival after inferior vena cava trauma. Am Surgeon 1999; 65(10): 976-81.

11. Buckman RF, Pathak AS, Badellino MM et al. Injuries of the inferior vena cava. Surg Clin North America 2001; 81(6): 1431-47.

12. Velmahos GC, Demetriades D, Toutouzas KG et al. Selective non-operative management in 1,856 patients with abdominal gunshot wounds: Should routine laparotomy still be the standard of care? Ann Surg 2001; 234(4): 395-403.

13. Daly KP, Ho CP, Person DL et al. Traumatic retroperitoneal injuries: Review of multidetector CT findings. Radiographics 2008; 28(6): 1571-90.

14. Khan IR, Jahromi AH, Khan FM et al. Nonoperative management of contained retrohepatic caval injury. Ann Vasc Surg 2012; 26(3): 420.e9-420.e12.

15. Renz BM, Feliciano DV. Unnecessary laparotomies for trauma: a prospective study of morbidity. J Trauma 1995; 38(3): 350-6.

16. Allen RE, Blaisdell FW. Injuries to the inferior vena cava. Surg Clin North America 1972; 52: 699-710. 
17. Bui TD, Mills JL. Control of inferior vena cava injury using percutaneous balloon catheter occlusion. Vasc Endovasc Surg 2009; 43(5): 490-3.

18. Angeles AP, Agarwal N, Lynd C. Repair of a juxtahepatic inferior vena cava injury using a simple endovascular technique. J Trauma Injury Infection Crit Care 2004; 56(4); 918-21.

19. Castelli $P$, Caronno R, Pifaretti $G$ et al. Emergency endovascular repair for traumatic injury of the inferior vena cava. EurJ Cardiothorac Surg 2005; 28(6): 906-8. 